\section{Gestão da interação Universidade-Empresa: o caso PUCRS}

Gabriela Cardozo Ferreira ${ }^{1}$ Alessandra Freitas Soria ${ }^{2}$ Lisiane Closs ${ }^{3}$

Resumo:A sociedade passou a exigir das universidades, além da formação de recursos humanos, atuação mais efetiva no processo de desenvolvimento econômico e social, reforçando a importância da contribuição da academia para o sistema de inovação. 0 presente artigo descreve uma pesquisa qualitativa realizada através de um estudo de caso único em uma universidade privada. Os resultados indicam que essa universidade está se adequando à nova realidade e vem realizando ações para internalizar conceitos como Inovação, Empreendedorismo e Universidade Empreendedora na comunidade acadêmica.

Palavras-chave: Universidade Empreendedora, Interação Universidade-Empresa, Transferência de Tecnologia, Rede de Inovação e Empreendedorismo.

\section{Introdução}

A mudança do paradigma da Sociedade Industrial para a Sociedade do Conhecimento coloca, no cerne das discussões, o conhecimento e sua gestão como fatores relacionados à capacidade competitiva de países e empresas (PLONSKY, 1999). No contexto atual, no qual se observam fenômenos como globalização e aceleração do desenvolvimento tecnológico, fatos que possibilitaram uma radical mudança na comunicação e no fluxo da informação, a inovação emerge como um elemento gerador de vantagem competitiva.

As profundas mudanças vividas nesse ambiente competitivo e dinâmico estabelecem novas exigências quanto à orientação e às formas de intervenção dos distintos agentes econômicos, governamentais, de ensino e da sociedade em geral. O conhecimento científico tem sido considerado um dos pilares que sustentam o desenvolvimento industrial, julgado por alguns como o insumo mais importante na geração de desenvolvimento econômico (ETZKOWITZ \& LEYDESDORFF, 2000).
Recebido:

04.08.2011

Aprovado:

11.05.2012

1. Professora do Mestrado e do Doutorado em Administração da Pontifícia Universidade Católica do Rio Grande do Sul (PPGAd/PUCRS). E-mail: gcferreira@ pucrs.br

2. Mestre em Administração pela Pontifícia Universidade Católica do Rio Grande do Sul (PUCRS)

E-mail: alessandra. soria@pucrs.br

3. Doutora em Administração pela Universidade Federal do Rio Grande do Sul (UFRGS) E-mail:

Icloss@terra.com. br 
Nesse ambiente, intensifica-se a interação Universidade Empresa (UE), gerando benefícios mútuos. A transferência de tecnologia (TT) manifesta-se como uma alternativa para as empresas promoverem a inovação e para as universidades obterem fontes complementares de recurso para a pesquisa (ETZKOWITZ, 2004). Emerge, assim, a preocupação com políticas internas e mecanismos de gestão que sustentem as atividades empreendedoras e inovadoras no habitat acadêmico.

Dado esse contexto, o presente estudo visa analisar a estratégia de uma universidade privada reconhecida como empreendedora e inovadora no Brasil no que diz respeito à interação com os demais atores da sociedade. Objetiva-se, especificamente, caracterizar a estruturas de apoio à inovação e ao empreendedorismo dessa universidade e identificar os mecanismos envolvidos nesse processo.

\section{A Universidade Empreendedora e a estrutura de apoio à inovação}

A Segunda Revolução Acadêmica somou às duas missões básicas da universidade, ensino e pesquisa, uma terceira, a colaboração com a economia, e tem como palavra-chave a capitalização do conhecimento (ETZKOWITZ \& LEYDESDORFF, 2000). Nesse contexto, Clark (2003) concebe o conceito de Universidade Empreendedora, como uma instituição ativa que faz mudanças na sua estrutura e no modo de reagir às demandas internas e externas. Clark (2003) realizou uma extensa pesquisa em cinco grandes universidades europeias, descritas como extremamente proativas nos seus esforços para reformar sua configuração. Esse estudo revelou cinco elementos comuns que identificam o caminho da transformação (CLARK, 2006; 2003):

1) renda diversificada da universidade: evidencia a ampliação de fontes de financiamento, tanto para a sustentabilidade da pesquisa como para a própria sustentabilidade da universidade;

2) capacidade fortalecida de administração: postura forte e clara da direção a ser seguida, aceita tanto pela administração central quanto pelos departamentos acadêmicos;

3) criação de novos mecanismos de apoio para as novas exigências, constituindo-se um entorno de desenvolvimento que consiste em centros de pesquisa não departamentais e programas de outreach;

4) núcleo acadêmico motivado, com perfil para assumir riscos, altamente proativo e empreendedor, que toma para si a necessidade de atualização permanente e busca novas soluções para problemas; 
5) cultura empreendedora integrada, representada por uma visão compartilhada, fator crítico para o sucesso da mudança e para a geração de uma perspectiva institucional.

No contexto de desenvolvimento de uma Universidade Empreendedora, destaca-se a importância das mudanças que a universidades vêm realizando em suas estruturas e mecanismos para atender às demandas originadas pela interação UE. A interação UE é uma relação de aprendizado interativo e inovador, porém, simultaneamente, envolve riscos de tensão e conflitos (LEVY, ROUX \& WOLF, 2009). Se a interface não for bem gerenciada, tanto a empresa quanto a universidade, aprofundarão frustrações recíprocas. Dessa forma, as universidades devem criar estruturas internas para promover e coordenar a interação UE, estabelecendo estratégias para articular ensino, pesquisa e extensão com a sociedade.

Devido ao grande número de formas de interação com atores externos, ressaltase a importância dos fatores ligados às estruturas de apoio e aos mecanismos de gestão por elas desenvolvidos que podem facilitar a interação UE, bem como políticas claras da universidade que incentivem uma cultura de depósito de patentes (AMADEI \& TORKOMIAN, 2009; GUARNICA \& TORKOMIAN, 2009).

Mudanças nas políticas internas e nas práticas organizacionais das universidades podem facilitar e aumentar o fluxo da interação e a TT com empresas (FRIEDMAN \& SILBERMAN, 2003). Um clima empreendedor tem um impacto significativo e positivo sobre todas as produções da TT universitária (RASMUSSEN, MOEN \& GULBRANDSEN, 2006). Além disso, uma missão clara do escritório de TT (LINK \& SIEGEL, 2005; MAYA, 2008) é um dos fatores mais impactantes no número de acordos para licenciamento e de invenções avaliáveis para licenciamento. Na realidade brasileira, estruturas específicas têm sido desenvolvidas pela universidade para auxiliá-las no processo de cooperação (SEGATTO-MENDES \& ROCHA, 2005; GARNICA \& TORKOMIAN, 2009). Essas estruturas de interface são mecanismos institucionais desenvolvidos para promover e facilitar a cooperação (PLONSKI, 1999), tais como: escritórios de transferência de tecnologia vinculados à Reitoria das Universidades; fundações conveniadas; estruturas empresariais com a missão de interagir com o segmento acadêmico; entidades de pesquisa sem fins lucrativos; entidades tecnológicas; entidades terceirizadas; rede de escritórios regionais vinculados ao governo local.

Na prática, observa-se, em várias universidades, a criação de diversos órgãos que têm como missão a ordenação da atividade acadêmica da produção e da TT (TERRA, 2001). Esses sistemas de comercialização podem incluir desde iniciativas de motivação e educação, até suportes específicos para a comercialização de projetos, como centros de inovação, incubadoras e fundos de capital 
semente (RASMUSSEN et al., 2006). Em muitos casos, diferentes atores se envolvem sozinhos ou em colaboração: a própria universidade, agências públicas, organizações não governamentais e companhias privadas (ibidem).

Os Parques Tecnológicos e as incubadoras de empresas nas universidades são também importantes intermediários na TT UE. Reduzem custos relativos à conversão de achados científicos em produtos ou processos, além de propiciar a transferência de conhecimento tácito entre pesquisadores e profissionais das empresas, favorecendo o sucesso dos inventos (AGRAWAL, KAPUR \& MCHALE, 2008). Nos centros de pesquisa cooperativa encontrados nessas estruturas, empresas de um mesmo setor se associam a uma universidade de alta qualificação em pesquisas daquele setor (SBRAGIA et al., 2006).

É importante ressaltar que tais estruturas e mecanismos deverão vir acompanhados de profissionais formados fora do escopo dos cursos de bases tecnológicas. Esses profissionais deverão ter noções de marketing, de proteção do conhecimento, de prospecção tecnológica, de avaliação do negócio tecnológico e, principalmente, ser interlocutores entre os agentes envolvidos no processo de integração UE. Destaca-se, ainda, uma convergência entre as atividades das estruturas de apoio, como os escritórios de TT e as incubadoras e parques tecnológicos, ficando a velocidade do processo dependente do amadurecimento das atividades de desenvolvimento tecnológico da universidade (LAHORGUE, 2005).

\section{Método}

Dado o objetivo deste trabalho, optou-se por uma investigação que permitisse analisar a estratégia da Universidade em sua interação com demais atores da sociedade, sobretudo no que tange à interação UE. Segundo Godoy (1995), assim como o ambiente natural surge como fornecedor direto de dados, quem realiza as pesquisas é visto como instrumento fundamental para a obtenção de informações que somente as pessoas em contato com o fenômeno podem oferecer. Sendo assim, realizou-se uma pesquisa de cunho qualitativo exploratório, adotando-se a estratégia de estudo de caso único.

Inicialmente, foi realizada a identificação de uma instituição com as características de uma universidade empreendedora (CLARK, 2003). Escolheu-se, então, a Pontifícia Universidade Católica do RS - PUCRS, já que esta possui estruturas de apoio bem definidas para interagir com organizações externas à universidade, articuladas em uma Rede de Inovação e Empreendedorismo, a INOVAPUC. Para uma melhor análise do objetivo deste estudo, buscou-se identificar interações entre a UE, investigando as estruturas de apoio à inovação e ao empreendedorismo, bem como os mecanismos envolvidos nesse processo. 
Inicialmente, foram identificados profissionais das estruturas de apoio à inovação envolvidos ativamente no processo de interação com empresas. Nesse sentido, foram entrevistados os gestores do Escritório de Transferência de Tecnologia (ETT) e da Agência de Gestão Tecnológica (AGT) e um consultor externo especializado em transferência de tecnologia.

Através da indicação dos gestores, foram selecionados integrantes de grupos de pesquisa e empresas, envolvidos em diferentes formas (ou conjunto de formas) de TT. As entrevistas com esses integrantes possibilitou uma análise da estratégia da Universidade em relação à interação com os demais atores da sociedade, sobretudo os ligados à universidade e empresas, conforme Quadro 1. O total de entrevistados foi 17 , compreendendo pesquisadores, gestores de inovação da PUCRS e equipes de empresas.

Quadro 1 - Relação das interações estudadas e entrevistados

\begin{tabular}{|l|l|l|}
\hline $\begin{array}{l}\text { INTERAÇÕES ESTU- } \\
\text { DADAS }\end{array}$ & № & ENTREVISTADOS \\
\hline Licenciamento de \\
patente & 4 & $\begin{array}{l}\text { Professor Doutor, vinculado à Faculdade de Química. } \\
\text { Professor Doutor, vinculado à Faculdade de Ciências Biológicas. } \\
\text { Professor Doutor, vinculado à Faculdade de Ciências Biológicas. } \\
\text { Coordenador de Pesquisa Clínica de um Laboratório Farmacêutico }\end{array}$ \\
\hline Pesquisa Conjunta & 7 & $\begin{array}{l}\text { Coordenador do Centro de Microgravidade } \\
\text { Gestor de Inovação de empresa do setor de equipamentos médico-hospitalares }\end{array}$ \\
\hline Financiamento de & 8 & Diretor de Instituto de Pesquisa Biomédica \\
Pesquisa & 14 & Administrador de um banco de células-tronco \\
\hline Spin-off & 9 & $\begin{array}{l}\text { Diretor de Centro de Pesquisa em Biologia em Molecular e Funcional } \\
\text { Pesquisador do Centro de Pesquisa em Biologia Molecular e Funcional } \\
\text { Sócio de uma empresa baseada no P\&D de insumos biotecnológicos }\end{array}$ \\
\hline Patente Conjunta & 11 & $\begin{array}{l}\text { Professor Doutor, vinculado a Faculdade de Engenharia } \\
\text { Gerente-Geral de uma empresa do setor de equipamentos médico-hospitalares } \\
\text { Gestor de Projetos de empresa do setor de equipamentos médico-hospitalares }\end{array}$ \\
\hline
\end{tabular}

Fonte: os autores (2011)

A coleta de dados foi realizada através da análise de documentos e de entrevistas em profundidade, cujo roteiro foi estruturado com base na revisão de literatura e, posteriormente, validado por três especialistas na área da Universidade. 
Os dados secundários foram obtidos através do acesso a sites e da análise de documentos, como contratos, portarias, entre outros. Os dados coletados foram analisados mediante a técnica de análise de conteúdo, que consiste em uma estratégia de pesquisa para descrever, de modo objetivo, sistemático e quantitativo, o conteúdo efetivo de uma comunicação (BARDIN, 1977).

\section{Análise e discussão dos resultados}

\section{A Pontifícia Universidade Católica do Rio Grande do Sul - PUCRS}

Ao longo dos últimos anos, várias ações foram desenvolvidas pela PUCRS para internalizar conceitos como Inovação, Empreendedorismo e Universidade Empreendedora. A fim de fortalecer, disseminar, dar suporte a esses conceitos e articular as unidades da universidade, foi criada a Rede de Empreendedorismo e Inovação da PUCRS - INOVAPUC. Para Audy e Ferreira (2006), a inovação se associa a todo processo de busca do novo que agregue valor à Universidade e, consequentemente, à sociedade. Já o empreendedorismo se relaciona com a geração de oportunidades a partir das inovações, com mudança das condições vigentes, a partir de novos recursos ou novas maneiras de utilizar recursos disponíveis (ibidem).

Nesse sentido, salienta-se o estabelecimento de políticas institucionais e estruturas de apoio pela alta administração da PUCRS. O empreendedorismo e a inovação estabelecidos entre seus pilares de gestão, expressos em seu estatuto, geram um ambiente acadêmico favorável à TT e à interação com empresas, aspecto valorizado pelos entrevistados e destacado por Santos e Solleiro (2006). A importância das políticas das universidades é também assinalada no contexto de países europeus que estão, como o Brasil, buscando melhorar a sua performance nesse processo (CALDERA \& DEBANDE, 2010; BALDINI et al., 2007).

A atitude da administração superior da universidade em investir na interação com organizações externas, sobretudo com empresas e governo, é percebida como um facilitador para os pesquisadores, tal como atestam outros autores (FRIEDMAN \& SILBERMAN, 2003; GARNICA \& TORKOMIAN, 2009). Conforme o entrevistado 8, Diretor do Instituto de Pesquisas Biomédicas:

Eu ouvi das palavras do Reitor que a PUCRS está absolutamente aberta a associações/parceiras/colaborações com empresas. Isso hoje acontece em universidades do mundo inteiro e traz muito a questão da autossustentabilidade. 
É condição sine qua non a liderança da administração superior no processo de aculturação do empreendedorismo e da inovação na Universidade e, consequentemente, no estabelecimento de políticas que possam dar suporte a essas ações. A importância da institucionalização da nova visão da Universidade e de uma postura forte e clara da direção sobre o caminho a seguir são corroboradas por Clark (2003) e Audy (2006).

A condição de pesquisa criada na PUCRS, a partir de sua visão de apostar na interação com a sociedade e, em especial, no relacionamento com empresas, é reconhecida como um facilitador, observado também por Amadei e Torkomian (2009) e Garnica e Torkomian (2009). Segundo o entrevistado 6: "Aqui você tem liberdade para produzir... aqui não é engessado".

Quando as universidades incorporam o conceito de universidade empreendedora e inovadora (CLARK, 2003), agregando ações como TT, é de extrema importância a valorização da pesquisa aplicada (BALDINI, GRIMALDI \& SOBRERO, 2007). Junto a isso, existe uma tendência de valorização das atividades externas do professor/pesquisador (OLIVEIRA \& FILION, 2008). Entre os critérios de seleção para contratação destes profissionais, poderão ser considerados, além da lógica do ensino, da pedagogia e da pesquisa, a experiência prática e o comportamento empresarial, bem como as lógicas da contribuição social e empresarial (ibidem).

Para articular as pesquisas de relevância da universidade com as demandas da sociedade, foi criada a Rede INOVAPUC, que congrega o conjunto de atores, ações, estruturas e mecanismos relativos ao processo de inovação e empreendedorismo da Universidade. Seu objetivo é promover o processo de inovação e empreendedorismo, articulando, para tal, todos os envolvidos no ensino, pesquisa e extensão. A INOVAPUC realiza um esforço multidisciplinar para buscar soluções e oferecer respostas às demandas da sociedade em termos de desenvolvimento econômico, social, ambiental e cultural. Dessa forma, tanto problemas identificados na sociedade podem dar origem ao desenvolvimento de pesquisas, quanto resultados e conhecimento já disponíveis na Universidade podem ser aplicados na solução de problemas existentes.

\section{Estruturas de apoio}

Não basta a vontade da administração superior, o desenvolvimento de estruturas de apoio à inovação é fundamental para criar condições para o desenvolvimento de um clima voltado ao empreendedorismo e à inovação (AUDY, 2006; BALDINI, GRIMALDI \& SOBRERO, 2007). Essas estruturas se constituem em espaços específicos de suporte para promover e facilitar atividades de cooperação 
que envolvem distintos mecanismos de gestão, de acordo com suas características.

A criação dos ambientes de apoio a inovação e ao empreendedorismo que estimulam e viabilizam a interação com o setor privado (AUDY, 2006) transmitem segurança ao pesquisador em participar de interações e atividades incomuns na sua rotina acadêmica. As estruturas de apoio são descritas a seguir:

\subsection{Agência de Gestão Tecnológica - AGT}

É a unidade responsável pela gestão das relações entre a PUCRS, as empresas e o governo no âmbito dos projetos de P\&D. Seu papel é de captar demandas da sociedade e canalizar respostas a elas geradas na universidade. A AGT é um dos principais contatos com as oportunidades que existem no mercado, tanto em relação às demandas para o desenvolvimento de projetos em conjunto com empresas, como para identificação de fontes de financiamento para a pesquisa na Universidade.

De modo geral, nas interações estudadas que apresentaram projetos de P\&D conjuntos, houve um grande envolvimento da AGT, desde a identificação de parceiros, preenchimentos de editais, negociação dos termos de parceria e administração dos contratos ao longo de todo o processo de TT.

Desse modo, quando analisado o apoio oferecido às relações com empresas e governo, foi identificado que essa estrutura preenche a lacuna existente entre esses atores. A AGT facilita a comunicação e cuida dos procedimentos necessários para o bom andamento da interação, sejam questões legais, aquisição de equipamentos e insumos, contratação de recursos humanos, preenchimento de formulários, negociação, entre outras questões transversais a todos os projetos e que não fazem parte do objetivo final da pesquisa. Conforme o entrevistado 2:

(...) A AGT possui todo um manual, que regra as relações (...). Trabalha (...) para que haja mecanismos facilitadores das ações e atividades. Então ele [o pesquisador] tem que, no máximo, assinar aquilo, verificar e assinar, se está de acordo.

Essa fala corrobora o publicado por Segatto-Mendes e Sbragia (2002), destacando que, entre as atribuições das estruturas de apoio, estariam aspectos da cooperação, como arrecadação, repasse e administração de recursos, divulgação das linhas de pesquisa da universidade, contato com as empresas parceiras potenciais, facilitação e manutenção da comunicação entre as partes.

O entrevistado 10 relata que a sua empresa 
(...) tem encontrado na AGT não um corpo de funcionários que faz a mera administração da interação universidade e empresa (...) e sim uma gestão estratégica de uma relação que está sendo construída.

A AGT, dentro do seu escopo de atuação, também pode motivar a interação UE. Por iniciativa do gestor da TT da AGT, que conhecia o antigo gestor de uma empresa, em um contato informal, revelou-se a possibilidade de um desenvolvimento conjunto de um desfibrilador. Conforme este gestor:

(...) eu os procurei e disse: olha, nós temos a oportunidade de transformar a empresa (...) Porque eles trabalhavam dentro de um modelo de cópia de coisas até ultrapassadas e eu disse: quem sabe vocês criam o próprio conhecimento e não ficam tão dependentes?

A AGT também auxilia as empresas a encontrar o parceiro ideal dentro dos inúmeros grupos de pesquisa da PUCRS. Nas palavras do entrevistado 2:

(...) às vezes as pessoas ainda não têm conhecimento mais abrangente dessas relações, que eventualmente a AGT pode ter, então começam a fazer sinapses, ligar pesquisador com empresa, ver um Edital FINEP e tentar juntar esse grupo.

Os depoimentos apresentados revelam o papel articulador da AGT que pode, através de sua expertise, motivar as relações das empresas com a universidade, utilizando fomentos disponíveis, em especial os aportados pelo governo.

\subsection{Escritório de Transferência de Tecnologia - ETT}

O ETT foi criado a partir da necessidade percebida pela PUCRS de proteger o seu patrimônio intelectual e promover a transferência dos resultados de pesquisa para o setor produtivo. Esse escritório tem por objetivos: estabelecer e promover a política institucional de propriedade intelectual (PI) e TT; implementar e divulgar os procedimentos necessários à proteção dos resultados de pesquisas realizadas nas diferentes unidades da PUCRS, bem como os relacionados à TT através da comercialização de ativos, protegidos ou não, de propriedade da Universidade.

Mecanismos de divulgação e a disseminação da cultura da PI são essenciais para o aumento da produção tecnológica nas universidades. Na PUCRS, essa tarefa é realizada pelo ETT, em fluxo contínuo, já que é praticamente inviável atingir toda a comunidade acadêmica de uma única vez. O resultado dessa atividade 
pôde ser identificado nas interações analisadas. Através de cursos promovidos pelo ETT, os pesquisadores iniciaram seu contato com as patentes, realizando atividades como pesquisa em banco de dados e análise do que pode ser patenteado. Pelo conhecimento do que já existia em relação às suas pesquisas, um pesquisador (entrevistado 4), por exemplo, buscou uma ideia inovadora, descrevendo algo que ainda não estava revelado em patentes:

O que eu vi foi uma oportunidade de mercado, eu li praticamente vinte patentes com trezentas folhas. E eu comecei a entender como se escreve uma patente, como é que se escondem os dados lá dentro (...) ele esqueceu isso, eu vou para bancada e faço.

Os serviços prestados pelo ETT são muito bem avaliados pelos entrevistados, como catalisador do processo de TT, deixando o pesquisador mais focado na pesquisa em si e não em questões burocráticas. Plonski (1999) conceitua essas estruturas de interface como mecanismos institucionais desenvolvidos para promover e facilitar a cooperação. A fala do entrevistado 6 ilustra essa questão: "se eu tiver que aprender a escrever uma patente, não sai. Procurar em banco de dados de patentes? Pode esquecer, não tenho tempo".

Esta afirmação ratifica a necessidade de criação de estruturas que facilitem o processo de TT, tornando-o factível para os pesquisadores, fato corroborado por Santana e Porto (2009). Com a limitação de tempo e a adição de processos diferentes daqueles da rotina do pesquisador, seria muito difícil a adesão em atividades de TT e interação com a empresa sem estruturas que gerenciassem as questões não ligadas diretamente à pesquisa, mas inerentes ao processo que envolve a interação com uma organização externa.

Em relação às empresas que interagem com a PUCRS, o fato de a universidade possuir uma patente, para o laboratório farmacêutico pesquisado, foi o grande facilitador para todo o processo, pois esse é um requisito imprescindível para a indústria farmacêutica. Nesse caso, além de ter a patente, o produto patenteado tem um alto potencial, com uma literatura científica que confirma isso e indicações terapêuticas importantes. Link e Siegel (2005) destacam como fator importante para a determinação do número de acordos para licenciamento o número de invenções avaliáveis para licenciamento, o que inclui o potencial de mercado das patentes. Esse fato corrobora a importância da pesquisa realizada na Universidade e da disseminação da cultura de patenteamento junto aos pesquisadores.

O entrevistado 9 considera o ETT como estrutura promotora e facilitadora do processo de interação e de TT com sua empresa. Segundo esse empresário, 
que interage com outras universidades no país, poucas universidades possuem um ETT como o da PUCRS. De acordo com o pesquisado, "a PUCRS tem fácil acesso, o pessoal é ágil, bastante organizado, bastante proativo, foi bem fácil".

As percepções do Entrevistado 9 reforçam a observação de Rasmussen, Moen e Gulbrandsen (2006), que afirmam que um ambiente empreendedor impacta positivamente sobre todas as produções da TT. Maya (2008) destaca que o compromisso mútuo para obter os resultados esperados e o estabelecimento de canais e mecanismos de comunicação impactam de forma positiva na TT.

O entrevistado 10 destaca, ainda, que o ETT foi identificado como estrutura importante para sua empresa, no auxílio em uma busca em banco de patentes. De forma ágil, o ETT mapeou todo o processo de uma determinada tecnologia, o que gerou alternativas à empresa para o desenvolvimento do produto.

\subsection{Instituto de Pesquisa e Desenvolvimento - IDÉIA}

O IDÉIA é uma unidade de apoio à pesquisa que dispõe de infraestrutura laboratorial, espaço físico para hospedagem de projetos de pesquisa de diversas unidades acadêmicas, atuando como incubadora de projetos e desenvolvimento de protótipos. Atua como uma área interdisciplinar, tendo por objetivo a pesquisa e o desenvolvimento.

Uma das interações analisadas teve seu projeto incubado no IDÉIA, entre os anos de 2005 e 2008. Esse período possibilitou o amadurecimento do projeto e o desenvolvimento do protótipo. Todo o aprendizado referente ao equipamento foi desenvolvido do zero, a empresa detinha uma parte do conhecimento, mas a tecnologia foi aprendida e desenvolvida na universidade. É importante ressaltar que o aluno envolvido com o projeto de pesquisa é, atualmente, o gestor de projetos da empresa parceira.

\subsection{Parque Científico e Tecnológico da PUCRS - TECNOPUC}

O TECNOPUC atua como gestor das relações da Universidade com as empresas e entidades localizadas no parque ou com interesse em nele se localizar, sendo também responsável pelo relacionamento com as várias estâncias do governo e com entidades que congregam parques e incubadoras. Tem com objetivo atrair projetos de pesquisa científica e tecnológica desenvolvidos em conjunto com empresas, inserindo a PUCRS diretamente no processo de desenvolvimento técnico, econômico e social da região e do país. É uma estrutura de apoio às atividades de inovação e empreendedorismo, que faz parte de uma 
concepção da Universidade em investir na pesquisa, em talentos e na interação com a sociedade. Sendo assim, tem como critério básico para instalação da empresa no local a manutenção de projeto de P\&D com a universidade, gerando bolsas para alunos de graduação e pós-graduação.

Nas interações analisadas, o parque científico e tecnológico da universidade foi identificado como estratégico para o processo de interação com as empresas. Na visão do entrevistado 9, diretor de uma spin-off, o grande facilitador da sua interação com a Universidade é o TECNOPUC. O TECNOPUC abriga a empresa spin-off envolvida neste estudo, resultado de projetos de pesquisadores da universidade.

\subsection{RAIAR}

A RAIAR é uma incubadora de base tecnológica multissetorial. Seu objetivo é abrigar empresas nascentes, cujos produtos, processos e serviços sejam resultados de pesquisas aplicadas, oriundas de projetos de alunos, professores e funcionários da PUCRS e de empreendimentos embrionários de empresas estabelecidas no TECNOPUC. Por meio do processo de incubação, as empresas poderão desenvolver as atividades necessárias para colocar seus produtos e serviços no mercado. Para tal, a incubadora conta com o Serviço de Apoio a Gestão, que auxilia as empresas nas áreas de gestão empresarial, entre as quais comercialização, comunicação e marketing dos produtos.

2.6 Laboratórios Especializados em Eletroeletrônica, Calibração e Ensaios - LABELO

O LABELO atua diretamente na área de prestação de serviços tecnológicos, sendo especializado em calibrações e ensaios, focado na área de metrologia científica e industrial, acreditado aos organismos de competência nacional. Segundo o Entrevistado 9, seu cronograma de serviços é cumprido rigorosamente, sendo respeitados os prazos acordados com a empresa. Essa sinergia em relação à variável tempo não é comum nas interações UE, fato usualmente elucidado pela diferença cultural entre os atores.

2.7 Centro de Inovação - Cl e Núcleo Empreendedor - NE

O Centro de Inovação é resultado de uma parceria entre a PUCRS e a MICROSOFT, tendo como objetivo acelerar o uso de novas tecnologias, fomentando 
a indústria nacional de software e contribuindo para a elevação da competitividade desse segmento no cenário nacional e internacional. O mesmo atua diretamente na capacitação de alunos de ensino médio e de graduação, da PUCRS ou de outras universidades, operacionalizando uma iniciativa que busca a criação de novas empresas inovadoras na área de Tecnologia da Informação.

O Núcleo Empreendedor tem por objetivo desenvolver ações relativas ao estímulo ao empreendedorismo na comunidade acadêmica. Desenvolve atividades, tais como palestras e eventos relacionados ao tema, programas de capacitação, identificação e desenvolvimento de competências para o empreendedor, bem com o apoio na área de gestão. Através de um concurso chamado Torneio Empreendedor, o NE busca despertar e estimular o espírito empreendedor dos alunos de graduação e pós-graduação. Desde sua operação, em 2007, seis equipes participantes do Torneio instalaram suas empresas na Incubadora RAIAR.

\section{Considerações Finais}

A análise de interações da universidade com empresas descritas neste estudo permitiu a identificação de aspectos relacionados às estratégias da PUCRS para estabelecer uma relação eficaz entre a academia e o meio empresarial. Enfatizase, primeiramente, a importância das diretrizes estabelecidas pela universidade para incentivar a inovação e o empreendedorismo.

As estruturas de apoio mostraram-se igualmente essenciais na interação com as empresas, como importantes mecanismos que assumem as atividades não ligadas diretamente à pesquisa, que despenderiam muito tempo do pesquisador e diminuiriam a adesão dos docentes em projetos de parceria com empresas e ações de TT. Tais estruturas transmitem, ainda, segurança para que as empresas possam formar parcerias de médio/longo prazo com a academia.

Afora isso, um interlocutor entre os atores possibilita minimizar as diferenças que porventura apareçam no andamento da interação UE. As estruturas de apoio são também importantes na identificação de parceiros, seja para o início dos projetos, na identificação das empresas e dos grupos de pesquisa que possam vir a interagir, seja ao longo do projeto na identificação de laboratórios para prestação de serviços tecnológicos.

O comportamento da universidade, ao se manter aberta e aparelhada com estruturas, políticas e atividades para desenvolver projetos em parceria com a sociedade, possibilita, ainda, a atração dos melhores talentos, tanto alunos quanto professores, na área de pesquisa e desenvolvimento. 
A fim de aprofundar a compreensão sobre o tema, sugere-se a investigação de fatores do macrocontexto que influenciam o processo de interação da Universidade Empresa, bem como a avaliação do impacto social e econômico dos resultados dessas interações. Estudos integrando outros atores e a análise das implicações das suas relações de poder nesse processo, sob uma perspectiva crítica, praticamente inexistentes nesta literatura, poderiam igualmente ampliar a compreensão sobre o tema, suprindo, simultaneamente, algumas das limitações deste trabalho.

Abstract: Society started to require from universities collaboration to foster its economic and social development, besides the development of human resources, reinforcing the academic contribution importance to the innovation system. This article describes a single case derived from qualitative data collected in a private university. Results show that this university is adapting to the new context and promoting activities to internalize, in the academic community, concepts as Innovation, Entrepreneurship and Entrepreneurial University.

Keywords: Entrepreneurial University, University-Industry interaction, Technology Transfer, Innovation Network, Entrepreneurship.

\section{Referências Bibliográficas}

AGRAWAL, A.; KAPUR, D.; MCHALE, J. "How do spatial and social proximity influence knowledge flows?" Journal of Urban Economics, v. 64, 2008, p. 258-269.

AMADEI, J. R. P.; TORKOMIAN, A. L. V. "As patentes nas universidades: análises dos depósitos das universidades paulistas". Ciência da Informação, v. 38, n. 2, 2009, p. 9-18.

AUDY, J. L. N. "Entre a Tradição e a Renovação: os desafios da Universidade Empreendedora", in J. L. N. Audy e M. C. Morosini (orgs.), Inovação e Empreendedorismo na Universidade. Porto Alegre: EDIPUCRS, 2006.

AUDY, J. L. N.; FERREIRA, G. C. “Universidade Empreendedora: a visão da PUCRS", in J. L. N. Audy e M. C. Morosini (orgs.), Inovação e Empreendedorismo na Universidade. Porto Alegre: EDIPUCRS, 2006.

BALDINI, N.; GRIMALDI, R.; SOBRERO, M. "To patente or not patente? A survey of Italian inventors on motivations, incentives and obstacles to university patenting". Scientometrics, v. 70, n. 2, 2007, p. 333-354.

BARDIN, L. Análise de Conteúdo. Lisboa: Edições 70, 1977. 
CALDERA, A.; DEBANDE, O. "Performance of Spanish universities in technology transfer: an empirical analysis". Research Policy, v. 39, 2010, p. 1160-1173.

CLARK, B. "Em busca da Universidade Empreendedora”, in J. L. N. Audy e M. C. Morosini (orgs.), Inovação e Empreendedorismo na Universidade. Porto Alegre: EDIPUCRS, 2006.

. Creating Entrepreneurial Universities. Oxford: IAU Press-Elsevier,

2003.

ETZKOWITZ, H. "The evolution of the entrepreneurial university". International Journal Technology and Globalization, v. 1, 2004, p. 64-77.

ETZKOWITZ, H.; LEYDESDORFF, L. "The dynamics of innovation: from National Systems and "Mode 2" to a Triple Helix of university - industry - government relations". Research Policy, n. 29, 2000, p. 109-123.

FRIEDMAN, J.; SILBERMAN, J. "University Technology Transfer: Do Incentives, Management, and Location Matter?" Journal of Technology Transfer, v. 28, 2003, p. 17-30.

GARNICA, L. A.; TORKOMIAN, A. L. V. “Gestão de Tecnologia em Universidades: uma análise do patenteamento e dos fatores de dificuldades e de apoio à transferência de tecnologia no Estado de São Paulo". Gestão \& Produção, v. 16, n. 4, 2009, p. 625-638.

GODOY, A. S. "A Pesquisa qualitativa: tipos fundamentais". Revista de Administração de Empresas, v. 35, n. 3, 1995, p. 20-29.

LAHORGUE, M. A. "Managing Relations with Industry: the case of Brazilian Universities". Higuer Education Mangement and Policy, v. 17, n. 2, 2005, p. 127-137.

LEVY, R.; ROUX, P.; WOLFF, S. "An analysis of science-industry collaborative patterns in large European University". Journal of Technology Transfer, v. 25, 2009, p. 111-133.

LINK, A.; SIEGEL, D. "Generating Science-based Growth: an econometric analysis of the impact of organizational incentives on university-industry technology transfer". European Journal of Finance, v. 11, 2005, p. 169-182.

MAYA, D. R. "El Processo de transferencia de tecnología" in J.L Solleiro e R. Castañón (orgs.), Gestión Tecnológica: conceptos y prácticas, México: PyV, 2008.

OLIVEIRA, J. B.; FILION, L. J. "Modelo Sinérgico de Pesquisa Subsidiada: transferência de tecnologia, criação de empresas e inovação". Revista de Administração e Inovação, v. 5, n. 1, 2008, p. 53-66. 
PLONSKI, G. A. "Cooperação universidade-empresa: um desafio gerencial complexo". Revista de Administração, v. 34, n. 4, 1999, p. 5-12.

RASMUSSEN, E.; MOEN, O.; GULBRANDSEN, M. "Iniciatives to promote commercialization of University Knowledge". Technovation, v. 26, 2006, p. 518-533.

SANTANA, É. E. de P.; PORTO, G. S. "E agora, o que Fazer com Essa Tecnologia? Um Estudo Multicaso sobre as Possibilidades de Transferencia de Tecnologia na USP". Revista de Administração Contemporânea, v. 12, n. 3, 2009, p. 410-429.

SANTOS, M. E. R.; SOLLEIRO, J. L. "Relações Universidade - Empresa no Brasil: diagnósticos e Perspectivas". in J. L. N. Audy e M. C. Morosini (orgs.), Inovação e Empreendedorismo na Universidade. Porto Alegre: EDIPUCRS, 2006.

SBRAGIA, R.; STAL, E.; CAMPANÁRIO, M. de A.; ANDREASSI, T. Inovação: Como vencer esse desafio empresarial. 1ed. São Paulo: Clio, 2006.

SEGATTO-MENDES, A. P.; SBRAGIA, R. O processo de cooperação universidadeempresa em universidades brasileiras". Revista de Administração da USP, v. 37 n. 4, 2002, p. 58-71.

SEGATTO-MENDES, A. P.; ROCHA, K. C. "Contribuições da teoria de agência ao estudo dos processos de cooperação tecnológica universidade-empresa". Revista de Administração, v. 40, n. 2, 2005, p. 172-183.

TERRA, B. A Transferência de Tecnologia em Universidades Empreendedoras Um caminho para a inovação tecnológica. Rio de Janeiro, Qualitymark, 2001. 